\title{
Wearable Monitoring for Mood Recognition in Bipolar Disorder Based on History-Dependent Long-Term Heart Rate Variability Analysis
}

\author{
Gaetano Valenza, Member, IEEE, Mimma Nardelli, Antonio Lanatà, Member, IEEE, Claudio Gentili, Gilles Bertschy, \\ Rita Paradiso, and Enzo Pasquale Scilingo, Member, IEEE
}

\begin{abstract}
Current clinical practice in diagnosing patients affected by psychiatric disorders such as bipolar disorder is based only on verbal interviews and scores from specific questionnaires, and no reliable and objective psycho-physiological markers are taken into account. In this paper, we propose to use a wearable system based on a comfortable t-shirt with integrated fabric electrodes and sensors able to acquire electrocardiogram, respirogram, and body posture information in order to detect a pattern of objective physiological parameters to support diagnosis. Moreover, we implemented a novel ad hoc methodology of advanced biosignal processing able to effectively recognize four possible clinical mood states in bipolar patients (i.e., depression, mixed state, hypomania, and euthymia) continuously monitored up to $18 \mathrm{~h}$, using heart rate variability information exclusively. Mood assessment is intended as an intrasubject evaluation in which the patient's states are modeled as a Markov chain, i.e., in the time domain, each mood state refers to the previous one. As validation, eight bipolar patients were monitored collecting and analyzing more than $400 \mathrm{~h}$ of autonomic and cardiovascular activity. Experimental results demonstrate that our novel concept of personalized and pervasive monitoring constitutes a viable and robust clinical decision support system for bipolar disorders recognizing mood states with a total classification accuracy up to $95.81 \%$.
\end{abstract}

Index Terms-Autonomic nervous system (ANS), bipolar disorder, heart rate variability (HRV), mood recognition, pervasive monitoring, wearable systems.

\section{INTRODUCTION}

W EARABLE systems for physiological monitoring have been gaining large interest over the last decade in

Manuscript received May 14, 2013; revised October 9, 2013 and August 14, 2013; accepted October 31, 2013. Date of publication November 11, 2013; date of current version September 2, 2014.

G. Valenza, M. Nardelli, A. Lanatà, and E. P. Scilingo are with the Department of Information Engineering and with the Research Centre "E. Piaggio," Faculty of Engineering, University of Pisa, 56126 Pisa, Italy (e-mail: g.valenza@ieee.org; mimma.nardelli@for.unipi.it; a.lanata@ centropiaggio.unipi.it; e.scilingo@ centropiaggio.unipi.it).

C. Gentili is with the Department of surgical, medical, molecular, and critical area pathology, section of psychology, University of Pisa, 56100 Pisa, Italy (e-mail: claudio.gentili@med.unipi.it).

G. Bertschy is with the Department of Psychiatry and Mental Health, Strasbourg University Hospital, INSERM U1114, University of Strasbourg, F-67000 Strasbourg, France (e-mail: gilles.bertschy@chru-strasbourg.fr).

R. Paradiso is with the Smartex srl, Navacchio 56023, Italy (e-mail: rita@smartex.it).

Color versions of one or more of the figures in this paper are available online at http://ieeexplore.ieee.org.

Digital Object Identifier 10.1109/JBHI.2013.2290382 research and commercial fields [1]-[27]. The key benefits introduced by these wearable systems include their small size, lightness, low-power consumption and, of course, wearability. Major applications of such systems are related to medicine and healthcare allowing for outpatient care and enhancing the quality of life for chronic disease patients, and preventing unnecessary hospitalizations. Long-term monitoring through wearable monitoring systems is a challenging task because of the many issues involved such as movement artifact rejection, comfort, and power consumption. In order to meet this challenge, long-term monitoring through multiple sensors are often recommended [1]. As a matter of fact, several systems have been recently developed for monitoring blood pressure [3], [4], cardiac activity [5], [6], respiration [7], eye tracking [2], multivariate physiological signs [6], [8]-[10], [23]-[26], electrodermal response [13], [14], physiological signs through antenna and UWB radar [11], [12], brain activity [15], as well as neurorehabilitation activity [16]. The state of the art on wearable systems for physiological monitoring can be found in these recent reviews [17]-[22].

In the abovementioned literature, some methods have proven better than others when it comes to developing wearable systems for physiological monitoring, namely those systems based on smart textiles used for autonomic nervous system (ANS) activity monitoring (e.g., see [6], [9], [13], [14], [23]-[27]). The major reason behind this choice is related to comfort and its insensitivity to artifacting events. Although signal dynamics coming from electroencephalograms (EEG) provide direct measurements of brain activity and, thus, are more effective in applications such as monitoring of mental disorders, peripheral measurements can also provide useful information. Indeed, both EEG and ANS dynamics are affected by a pathological mental status [9], [28]-[32]. It has been demonstrated how affective elicitations lasting longer than $6 \mathrm{~s}$ allow the prefrontal cortex to encode the stimulus information and to transmit it to other areas of the central autonomic network down to the brainstem, thus producing a context appropriate response [33]. Moreover, cortisol hormone levels are affected in mood disorders [34]-[36]. Finally, the parasympathetic ANS is anatomically connected to central nervous system activity. As a counter-proof, vagus nerve stimulation has been successfully used for the treatment of resistant depression [37].

Recently, we have proposed a preliminary evaluation of a multiparametric wearable platform for physiological/behavioral monitoring of mood fluctuation in bipolar patients [9]. The 
system has been developed in the frame of a European Project, Personalized monitoring SYstems for Care in mental HEalth (PSYCHE), whose goal was to find possible correlations between ANS patterns and mood swings over short- and longterm monitoring (see details in Section II). In this study, we present a part of the PSYCHE concept consisting of a wearable monitoring platform enriched with a novel and effective signal processing methodology able to recognize mood states using long-term cardiovascular dynamics and past mood labels. Given the experimental evidence over the past three decades on ANS and cardiovascular dynamics, we have chosen the heart rate variability (HRV) analysis [38], [39] as a noninvasive marker for a personalized and accurate management of patients affected by bipolar disorder.

\section{A. Background on Bipolar Disorders}

Bipolar disorder is very common in western population [40][43]. A recent worldwide survey in 11 countries has found an overall lifetime prevalence of $1 \%$ for the typical forms of bipolar disorder and $1.4 \%$ for milder subthreshold disorders [44].

From the clinical point of view, bipolar disorder is a chronic condition characterized by an impaired mood balance. Patients experience episodes of altered mood states ranging from depression with sadness, hopelessness (including suicidal ideation), loss of energy, anhedonia, and psychomotor retardation to mania characterized by euphoria or irritability, excessive energy, hyperactivity, hypertrophic self-esteem, reduction in the need of sleep, and psychomotor acceleration. The moderate form of mania is called hypomania (at the stage of mania, change in behavior is so obvious that hospitalization is needed). Accordingly, it is possible to identify four possible types of mood episodes associated with such a disease: depression (DP), mania (MN), hypomania (HY), and mixed state (MS), which corresponds to the simultaneous presence of depression and mania. Patients experiencing periods of relatively good affective balance are defined as staying in euthymic state (ES) or remission.

\section{B. Limits in the Diagnostic Approach of Bipolar Disorders}

The diagnosis of bipolar disorders and, more in general, of psychiatric pathological conditions is based on clinical evaluations through interviews and the evaluations of scores gathered by quantitative psychopathological rating scales only. Although these interviews are "structured" (i.e., questions and question order are established and defined in specific manuals) and high rates of consensus can be achieved among specialists (psychiatrists and clinical psychologists), the diagnosis is always based on clinician observation, the patient's subjective description, and on the physician's interpretation of such description. However, specific physiological or biochemical markers to be taken into account in current clinical practice are still missing. The most important diagnostic system is based on the criteria proposed by the diagnostic statistic manual of mental disorders (DSM-IVTR) [45] edited by the American Psychiatric Association. According to this manual, to be diagnosed with depressive episodes the patient must have five out of nine possible symptoms. Similar cutoffs are applied for the diagnosis of other episodes. In line with this approach, a patient who has had only four symptoms of depressive episodes is considered remitted (although partially remitted). These clearly can bring to biased interpretation and inconsistency [46]-[48].

To overcome these issues, research has been done on proposing biomarkers that consider sleep quality [49]-[51], circadian heart rate rhythms [52], [53], cortisol dynamics, [34]-[36], as well as ANS functionality [9], [28]-[32]. However, none of these studies has reached an acceptable level of accuracy for clinical use in order to forecast the clinical course in patients. In a recent study [9], we presented a multiparametric approach that was successfully applied as a decision support system for the diagnosis of bipolar disorder. We demonstrated that a singlevariable approach, as proposed by previous literature, is not sufficient to robustly characterize mood episodes [9]. Instead, a multiparametric and personalized approach, i.e., mood episodes are identified as an intrasubject analysis, is much more effective. However, limitations of such an approach were twofold: first, the temporal dynamics of a patient's mood episodes were not taken into account (patients passing from depression to euthymia through a mixed state would be evaluated as patients passing from mixed state to depression through euthymia); second, patients experiencing more than two mood states were assessed by performing comparative evaluations for each couple of mood states, i.e., two-class pattern recognition problem.

Here, we overcome such limitations describing a novel and effective signal processing methodology applied to HRV data gathered from bipolar patients, which significantly improved our previous results. More specifically, the major assumption of the method hereby proposed is that clinical mood state depends on the previous mood state. In fact, in current clinical practice, it is widely accepted that a specific psycho-pathological status is differently characterized by taking into account the clinical history of the illness.Accordingly, our personalized approach identifies mood states as an intrasubject analysis taking into account the temporal dynamics of the illness. From a signal processing point of view, patients' mood changes are modeled as a discrete-time stochastic process in which each recording, associated with a specific mood state, also depends on the previous state respecting the so-called Markov property [54]. Of note, computational architectures for modeling of human emotions were proposed as being based on the Markov's theory [55].We demonstrate that mood changes in bipolar disorders can be modeled as a Markov chain, in which each state is characterized by ANS-HRV features extracted over long periods of time (up to $18 \mathrm{~h}$ ). Multiclass recognition of these mood states achieved an accuracy as high as $99 \%$.

\section{THE PSYCHE SYSTEM AND THE WEARABLE MONITORING PLATFORM}

PSYCHE stands for PerSonalized monitoring sYstems for Care in mental HEalth and identifies a personalized, pervasive, cost-effective, and multiparametric system for the long-term and short-term acquisition of data gathered from patients affected by mood disorders [9], [56], [57]. It was designed and is currently used in the framework of a European project PSYCHE, 


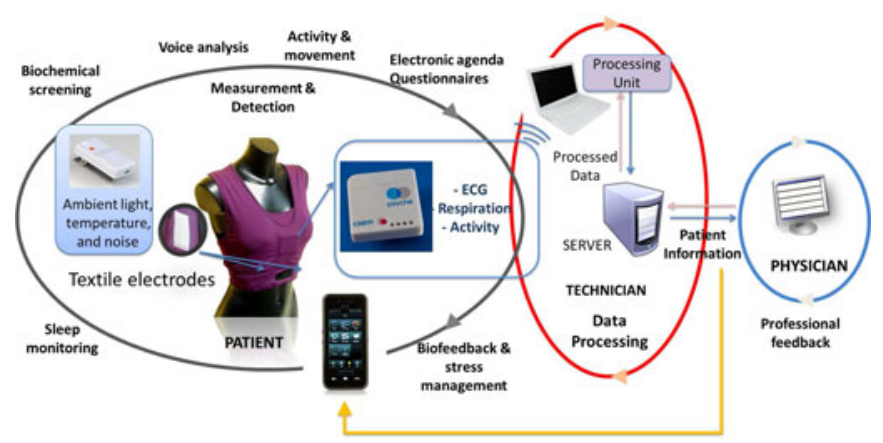

Fig. 1. Overview of the PSYCHE system as a global platform serving as decision support system for bipolar disorder management.

which is funded in the seventh framework programme (FP7). The PSYCHE system supports a novel approach for bipolar disease management based on the paradigm that quasi-continuous monitoring in a natural environment providing parameters, indices, and trends that will be used to assess mood status, support patients, predict and anticipate treatment response in its early phase, prevent relapse, and to alert physicians in case of a critical event. PSYCHE serves as a decision support system that fills the gap between research and clinical routine management of bipolar patients integrating the traditional clinical standard procedures of mood assessment with data coming from the pervasive system, which includes physiological signals, as well as biochemical and behavioral data. A centralized server performing data-mining procedures for mood evaluations is currently under development along with the implementation of a user-friendly patient interface (e.g., on a smartphone), and a professional web-based interface used by clinicians to administer evaluation questionnaires, look at physiological variable variations, communicate with patients, etc. The user-friendly device will also monitor environmental information such as light, temperature, and noise thereby completing the PSYCHE platform (see the PSYCHE concept of decision support system for bipolar disorder on Fig. 1). Several signals are taken into account for the patient's physiological monitoring such as voice [57], activity index, sleep pattern alteration [58], electrodermal response [56], respiration activity, and electrocardiogram (ECG) [9].

In this study, we used the core sensing system of the project, i.e., the PSYCHE wearable monitoring platform [9], whose technical specifications are reported in Table I. It was developed by Smartex s.r.l (Pisa, Italy) and consists of a comfortable sensorized t-shirt having dry textile-based electrodes that acquire the patient's ECG, a piezoresistive sensor to acquire the respiration signal, and a three-axial accelerometer to track movement. It is worth noting that the use of dry textile-based electrodes provides several advantages. First, the system is easy to use and allows to maximize comfort and to locate the sensors automatically. Second, a special multilayer structure increases the amount of sweat and reduces the rate of evaporation reaching electrochemical equilibrium between the skin and electrodes after a couple of minutes. Therefore, the signal quality [6] is remarkably improved and kept as constant as possible. If contacts
TABLE I

TeChNical SPECifications of the Wearable Monitoring Platform (PROVIDED BY SMARTEX S.R.L.)

\begin{tabular}{|c|c|}
\hline \multicolumn{2}{|c|}{ Characteristics } \\
\hline \hline Power supply & Litium battery (life up to 18 hours) \\
\hline Data storage & MicroSD card \\
\hline Data communication & Micro USB, bluetooth \\
\hline \multicolumn{2}{|c|}{ Electrocardiogram } \\
\hline Measurement principle & Bio-potentials on the thorax \\
\hline Sensors & Textile electrodes \\
\hline Number of leads & 1 \\
\hline Input auto configurable analog filter & $0.67 \mathrm{~Hz}$ to $40 \mathrm{~Hz}$ \\
\hline Analog-to-digital conversion & 16 bits \\
\hline Sampling rate & $250 \mathrm{~Hz}$ \\
\hline \multicolumn{2}{|c|}{ Respiration signal } \\
\hline Measurement principle & Piezoresistive method \\
\hline Range of electrical resistance & $20 \mathrm{k} \Omega$ to $10 \mathrm{M} \Omega$ \\
\hline Bandwidth & DC to $10 \mathrm{~Hz}$ \\
\hline Resolution & $12 \mathrm{bits}$ \\
\hline Sampling rate & $25 \mathrm{~Hz}$ \\
\hline
\end{tabular}

with the skin in not good, for reasons of size, the quality of the signals cannot be adequate for obtaining meaningful values. To avoid this problem, a preliminary check on the quality of the data is done using available shirts with different sizes before giving the system to the patients. The shirt was designed following both a female and male model and is made of elastic fibers that allow for tight adhesion to the user's body, piezoresistive fibers to monitor fabric stretching (and consequently respiration activity), and metallic fibers knitted to create fabric electrodes to monitor the ECG. These materials are knitted together and are fully integrated in the garment without any mechanical and physical discontinuity, creating areas with different functionalities. In this study, a comfortable t-shirt having two textile ECG electrodes integrated in the inner side of the front part, below the pectoral muscles in men and the breasts in women, and a textile piezoresistive sensor located between the electrodes, on the outer side, was used. The shirt is conceived to be similar in term of look and feel perception to any other ordinary underwear garment. The shirt has been designed taking into account the thermal comfort, both in the selection of the yarns and in the stitches structure, several zones with an open net have been inserted, and a polyamide yarn with antibacterial properties and a natural feeling has been used as a basic component. As a matter of fact, garments are made of commercial yarns, already tested (and certified) for contact with human skin, and can be easily washed and, in case, disinfected. The two ECG electrodes and a piezoresistive sensor are finally connected to the portable electronics, which is connected to the garment through a simple plug that can be easily unplugged when necessary.

In this study, we used ECG information coming from the PSYCHE wearable monitoring platform in order to extract the interbeat interval series (hereinafter RR), i.e., the series constituted by the distance of two consecutive peaks of the ECG in a patient as a noninvasive biomarker of ANS dynamics [38], [39]. While using the PSYCHE system, patients are asked to wear the sensorized shirt. They are free to perform daily activities at home or elsewhere, while the aforementioned physiological 


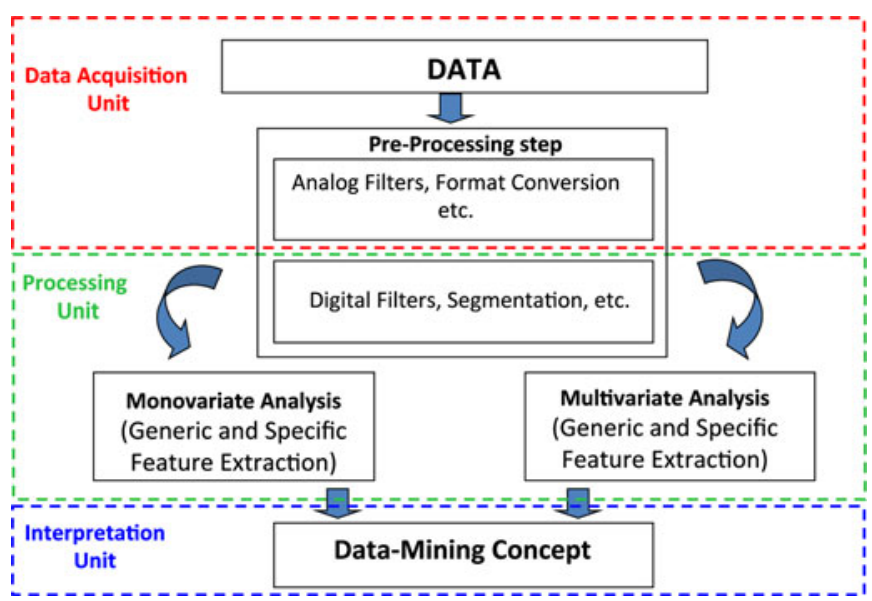

Fig. 2. Block scheme of the overall processing platform divided in three logical units for acquisition, processing, and interpretation.

signals are monitored and stored in a microSD card. After about $18 \mathrm{~h}$ of monitoring, patients are asked to take the system back to their physician, and the recorded data ae manually sent to a central database. As aforementioned, the final version of the PSYCHE system will provide an automatized storage process by means of a smartphone or tablet without the involvement of a technician.

\section{DATA ACQUisition, PROCESSING, AND INTERPRETATION}

Acquisition, processing, and interpretation procedures aim at extracting significant information from the acquired data in order to well define and characterize the patient's mood state. Such procedures are divided into three units: data acquisition, processing, and interpretation, whose general block scheme is shown in Fig. 2.

\section{A. Data Acquisition and Processing}

Data acquisition is fully implemented in the embodied electronic device of the wearable platform and includes also a part of the preprocessing step. In this unit, the analog ECG is acquired and conditioned by means of an instrumentation amplifier and filters. Then, the ECG is digitalized with a sample frequency of $250 \mathrm{~Hz}$ and stored into the microSD card for further analysis. The processing unit is responsible for extracting and analyzing features from the stored raw data collected as ASCII files. The ECG signal is prefiltered through a tenth-order band-pass finite impulse response filter with cutoff frequencies of $0.05-35 \mathrm{~Hz}$ approximated by the Butterworth polynomial. Considering that the patients wearing the sensorized shirt can move during the acquisition, and that textile electrodes could lose contact with the skin during body movement, an ad hoc algorithm for the automatic removal of movement artifacts has been applied [9]. To this aim, the maximum and minimum envelopes of the ECG filtered in the bandwidth from 0.1 to $4 \mathrm{~Hz}$ [59] are calculated. Afterwards, movement artifacts are detected by using simple statistical thresholds, i.e., 95th percentile, on the average envelope aforesaid, which the signal is considered affected by artifacts.
Further analyses such as R-peaks and feature extraction were performed in all of the available artifact-free, nonoverlapped time windows $W$ of $5 \mathrm{~min}$. Parts of the signals with artifacts together with consecutive artifact-free ECG signals having dynamics less than 5 min were discarded and not considered for further analysis. The $W$ of 5 min was chosen in order to fulfill the stationarity requirements in analyzing long-term RR series (see recommendations in [38] and [60]).

In RR series extraction, referring to the change of the beat interval corresponding to the $R$-peak, the well-known automatic algorithm developed by Pan-Tompkins [61] was adopted for automatic $R$-wave detection. Given the RR interval series, the analysis is performed by extracting significant features using the state of the art for assessing the autonomic regulation of the heart rate [38], [39]. In particular, standard parameters that are defined in the time and frequency domain and are correlated with the sympatho-vagal balance as well as nonlinear measures are taken into account. Time-domain features include the average and standard deviation values of the RR intervals, the square root of the mean of the sum of the squares of differences between subsequent NN intervals (RMSSD), and the number of successive differences of intervals that differ by more than $50 \mathrm{~ms}$ (expressed as a percentage of the total number of heartbeats analyzed, pNN50). Moreover, the triangular index was calculated as a triangular interpolation of the HRV histogram. All extracted features in the frequency domain were based on the power spectral density (PSD) of the HRV. An autoregressive (AR) model was used to estimate the PSD in order to provide better frequency resolution than in nonparametric methods. The optimal order $p$ was estimated according to the Akaike information criterion [62]. The Burg method was used to obtain the AR model parameters. The standard frequency-domain parameters were: very low frequency, this spectral component in general below $0.04 \mathrm{~Hz}$; low frequency (LF), ranging between 0.04 and $0.15 \mathrm{~Hz}$, and high frequency (HF), which is up to $0.4 \mathrm{~Hz}$. For each of the three frequency bands, the peak value corresponding to the frequency having maximum magnitude was also evaluated. Moreover, the LF/HF ratio was calculated in order to quantify sympathovagal balance and to reflect sympathetic modulations [38], [39].

Several nonlinear HRV measures were also extracted along with the standard morphological and spectral features [38], [39]. Even if the physiological meaning of these features is still unclear, they resulted to be an important quantifier of cardiovascular control dynamics mediated by the ANS [63]-[72]. Nonlinear measures are referred to as features extracted by means of the phase space (or state space). Once the phase space is estimated (by means of the so-called embedding procedure), the parameters that appears subject to an ANS modulation were evaluated. More specifically, correlation dimension [73], sample entropy, and approximate entropy [74], [75], features from the recurrence plot [76] by means of the recurrence quantification analysis (RQA) [77], and the detrended fluctuation analysis [78], [79] were evaluated. RQA was chosen to quantify the number and the duration of recurrences of the considered cardiovascular dynamical system. The following features were calculated [77]: 


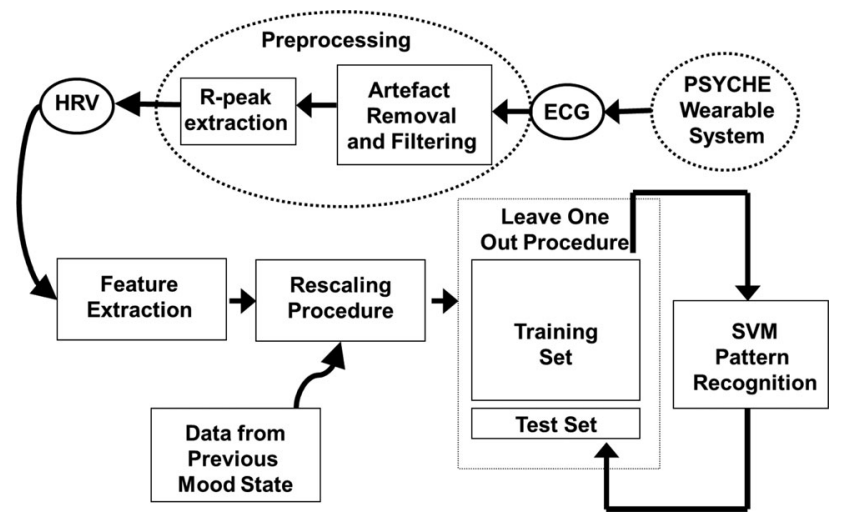

Fig. 3. Overall block scheme of the proposed mood recognition system.

recurrence rate, determinism, laminarity, trapping time, average diagonal line length, entropy, and longest diagonal line.

\section{B. Data Interpretation Through History-Dependent Analysis and Pattern Recognition}

Data interpretation is based on a personalized model in order to accurately associate and recognize the obtained parameters with the clinical mood status of the patient. Here, personalization is intended as intrasubject analysis. A logical scheme of the proposed mood recognition system, which also includes the previous steps, is shown in Fig. 3. Once all HRV features are extracted for each patient, the feature set related to the $k^{t h}$ acquisition can be defined as a multidimensional vector $X_{n k}\left(\mathrm{~T}_{\mathrm{m}}\right)$ representing $n$ features evaluated within the time window $T_{m}$. Therefore, $X_{n k}\left(\mathrm{~T}_{\mathrm{m}}\right)$ is a matrix of features consisting of $m$ rows (each of the rows corresponds to one of the time windows $T_{m}$ within the acquisition) and $n$ columns (each of the columns contains one of the HRV features). In order to consider the process of mood states such as the Markov chain, i.e., $\operatorname{Pr}\left\{X_{n k}=x \mid\left(X_{n(k-1)}=x_{k-1}\right)\right\}$, a simple rescaling procedure is applied. Specifically, for each column of the matrix $X_{n k}\left(\mathrm{~T}_{\mathrm{m}}\right)$, the matrix $Y_{n k}\left(\mathrm{~T}_{\mathrm{m}}\right)=\left[X_{n k}\left(\mathrm{~T}_{\mathrm{m}}\right)-\right.$ $\left.\operatorname{Median}\left(X_{n(k-1)}\left(\mathrm{T}_{\mathrm{m}}\right), m\right)\right] / \operatorname{MAD}\left(X_{n(k-1)}\left(\mathrm{T}_{\mathrm{m}}\right)\right)$ is calculated, where $\operatorname{MAD}(X)=\operatorname{Median}(|X-\operatorname{Median}(X)|)$. The quantity $\operatorname{Median}\left(X_{n(k-1)}\left(\mathrm{T}_{\mathrm{m}}\right), m\right)$ is intended as a vector of the median values of the features calculated through all the rows $m$ of the matrix $X_{n k}\left(\mathrm{~T}_{\mathrm{m}}\right)$, thus over all the time windows $T_{m}$ of the acquisition $k-1$.

This model is justified by the hypothesis that the transition from a clinical mood state to another is dependent on the past history of mood fluctuations. In other words, the current clinical status of a patient is influenced by the previous status, and therefore also the neurovegetative balance should be rescaled by a factor taking into account the previous clinical status. A simplified block scheme representing such a rescaling procedure over multiple mood states of a patient is shown in Fig. 4. The first observation of each patient was used exclusively to obtain the rescaling values useful for the characterization of the observation, which followed in time. This choice was justified by the fact that no preceding recordings were available.

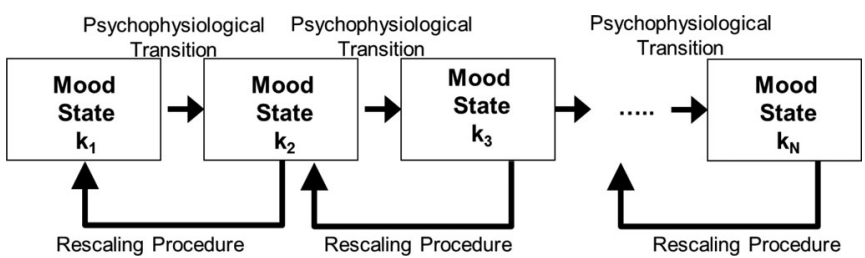

Fig. 4. Simplified block scheme over multiple mood states representing the Markov modeling of the mood recognition procedure.

The obtained feature set $Y_{n k}\left(\mathrm{~T}_{\mathrm{m}}\right)$ is taken as an input of the leave-one-out (LOO) procedure [80] applied on a support vector machine (SVM)-based pattern recognition [81]. More specifically, we used a nu-SVM $(\mathrm{nu}=0.5)$ having a radial basis kernel function with $\gamma=n^{-1}$. A mood label, given by psychological clinical assessment, was associated with each point in the feature space $Y_{n k}\left(\mathrm{~T}_{\mathrm{m}}\right)$. More specifically, the initial mood, diagnostic of the current bipolar episode, was determined by clinicians according to DSM-IV-TR criteria [45] and resulted in a diagnosis of current episode of depression, hypomania, or mixed state, which was the mood label at study entry. Then, its evolution toward another mood state (for instance transition from hypomania to mixed state) or an ES (remission of manic and depressive symptoms) was assessed using scores from quantitative psychopathological rating scales (for the scales and the threshold scores, see paragraph IV). This was a purely clinical evaluation that disregarded any physiological/biochemical reference analysis. The mood label associated with each patient's evaluation was assigned independently with respect to the previous ones. The focus on intrasubject analysis is mainly due to the small number of patients involved and is focused to find possible correlations between the pattern of physiological signals and mood fluctuations, which are much more interesting for the psychiatric community. In order to compare the proposed methodology with a standard approach, we evaluated the LOOSVM performance in discerning different mood patterns that are independently processed. In other words, the feature set belonging to a specific acquisition of a patient is taken as an input for the LOO-SVM classification without performing any rescaling procedure, i.e., without using information related to previous mood states. All of the classification results were expressed as recognition accuracy in detailed confusion matrices [82]. The generic element $r_{i j}$ of the confusion matrix indicated a percentage as to how many times a pattern belonging to class $i$ was classified as belonging to class $j$. A more diagonal confusion matrix corresponded to a higher degree of classification. All of the algorithms were implemented by using Matlab v7.2 endowed with two additional toolboxes for pattern recognition, i.e., LIBSVM [83] and a time series analysis toolbox [84].

\section{EXPERIMENTAL PROTOCOL AND RESUltS}

Besides the PSYCHE system and methodology of signal processing, an innovative aspect of this study is also represented by the experimental protocol undertaken. In the following paragraphs, the recruitment of eligible subjects, experimental procedures, and results are described in detail. 
TABLE II

Clinical Labels Associated With Each Patient DuRing EACH ACQUISITION

\begin{tabular}{|c|c|c|c|c|c|c|}
\hline ID & ACQ. 1 & ACQ. 2 & ACQ. 3 & ACQ. 4 & ACQ. 5 & ACQ. 6 \\
\hline \hline BP1 & HY & HY & HY & ES & ES & \\
\hline BP2 & HY & MS & HY & HY & & \\
\hline BP3 & HY & HY & HY & ES & ES & \\
\hline BP4 & DP & DP & DP & DP & DP & ES \\
\hline BP5 & DP & DP & HY & DP & HY & \\
\hline BP6 & HY & HY & HY & ES & ES & \\
\hline BP7 & DP & DP & ES & & & \\
\hline BP8 & MS & MS & DP & DP & DP & ES \\
\hline
\end{tabular}

Recruitment of eligible subjects: Patient recruitment was performed according to the following criteria.

1) Age between 18-65.

2) Presence of a mood episode at the moment of the recruitment of any polarity (depression, hypomania, mixed).

3) Low risk of suicidality (as assessed as no thoughts of death and no previous attempts).

4) No somatic or neurologic disorders that might be related to bipolar disorders (e.g., thyroid alterations).

5) Absence of cognitive impairment.

6) Absence of substance abuse disorders.

7) Necessity of a change in treatment (treatment change is defined as a augmentation of doses, introduction of or switch to new drugs, introduction of physical treatments).

8) Willingness of all patients to sign the informed consent for the PSYCHE project approved by the ethical committee of the University of Pisa and Strasbourg.

Following this criteria, 15 patients were recruited in the PSYCHE project, seven in Pisa, and eight in Strasbourg. Among these, we used for our study eight subjects for a total of 42 acquisitions (two from Pisa and six from Strasbourg) because they had more than one long-term acquisition and at least one mood change.

Details on the patients' acquisitions as well as mood state information are reported in Table II.

Experimental procedures: Patients BP1, BP2, BP3, BP4, BP5, and BP6 were recruited in the out-patient University clinic of Strasbourg, France. Patients BP7 and BP8 were recruited in high-intensity clinical facilities (psychiatric ward and day hospital) at the University Hospital of Pisa, Italy. The protocol planned a study entry visit when the patient was experiencing a depressive, hypomaniac, or mixed phase. Patients were studied with an average frequency of 2-3 times a month. Each patient was evaluated and monitored from the day of the hospital admission toward remission, i.e., until the reaching of an ES as long as such a condition was presented within three months after the first visit. In any case, in this study no more than six evaluations per patient were performed. All clinical states were evaluated by clinicians according to DSM-IV-TR criteria [45]. In this way, four possible clinical mood labels (depression, hypomania, mixed-state, and ES) were assigned. The mood label associated with each patient's evaluation was assigned independently with respect to the previous ones. ES, i.e., clinical remission was defined by having a score below threshold on a quantitative psychopathological
TABLE III

CONFUSION MATRIX OF SVM CLASSIFIER FOR BP1

\begin{tabular}{|c|c|c|c|}
\hline & Dataset & Hypomania & Euthymia \\
\hline \hline \multirow{2}{*}{ Hypomania } & Standard & $\mathbf{9 1 . 9 9}$ & 8.01 \\
\cline { 2 - 4 } & Markov & 88.64 & 11.36 \\
\hline \hline \multirow{2}{*}{ Euthymia } & Standard & 80.40 & 19.60 \\
\cline { 2 - 4 } & Markov & 29.20 & $\mathbf{7 0 . 8 0}$ \\
\hline \multirow{2}{*}{$\begin{array}{l}\text { Total Accuracies: } \\
\text { indicate the best correct classification results for each mood }\end{array}$}
\end{tabular}

state.

TABLE IV

CONFUSION MATRIX OF SVM CLASSIFIER FOR BP3

\begin{tabular}{|c|c|c|c|}
\hline & Dataset & Hypomania & Euthymia \\
\hline \hline \multirow{2}{*}{ Hypomania } & Standard & 96.70 & 3.30 \\
\cline { 2 - 4 } & Markov & $\mathbf{9 7 . 7 8}$ & 2.22 \\
\hline \hline \multirow{2}{*}{ Euthymia } & Standard & 64.44 & 35.56 \\
\cline { 2 - 4 } & Markov & 11.11 & $\mathbf{8 8 . 8 9}$ \\
\hline
\end{tabular}

Total Accuracies: Standard 66.13\%; Markov 93.33\%. Bold values indicate the best correct classification results for each mood state.

TABLE V

CONFUSION MATRIX OF SVM CLASSIFIER FOR BP2

\begin{tabular}{|c|c|c|c|}
\hline & Dataset & Hypomania & Mixed-State \\
\hline \hline \multirow{2}{*}{ Hypomania } & Standard & $\mathbf{9 7 . 0 3}$ & 2.97 \\
\cline { 2 - 4 } & Markov & 86.46 & 13.54 \\
\hline \hline \multirow{2}{*}{ Mixed-State } & Standard & 62.5 & 37.5 \\
\cline { 2 - 4 } & Markov & 3.75 & $\mathbf{9 6 . 2 5}$ \\
\hline
\end{tabular}

Total Accuracies: Standard 67.26\%; Markov 91.35\%. Bold

values indicate the best correct classification results for each mood state.

TABLE VI

CONFUSION MATRIX OF SVM CLASSIFIER FOR BP4

\begin{tabular}{|c|c|c|c|}
\hline & Dataset & Depression & Euthymia \\
\hline \hline \multirow{2}{*}{ Depression } & Standard & 98.53 & 1.47 \\
\cline { 2 - 4 } & Markov & $\mathbf{9 9 . 6 8}$ & 0.32 \\
\hline \hline \multirow{2}{*}{ Euthymia } & Standard & 83.91 & 16.09 \\
\cline { 2 - 4 } & Markov & 8.05 & $\mathbf{9 1 . 9 5}$ \\
\hline
\end{tabular}

Total Accuracies: Standard 57.31\%; Markov 95.81\%. Bold values indicate the best correct classification results for each mood state.

rating scale (for depressive symptoms, score below 8 on the 16item quick inventory of depressive symptomatology clinician rating and for manic symptoms score below 6 on the Young Mania rating scale). The same thresholds were also used to define a change in mood state. During the study, treatment choice remained at the discretion of the clinician as well as the change of treatment in case of lack of response.

The wearable system was given to the patients in the afternoon and collected after the morning. Patients receiving the PSYCHE wearable monitoring platform were asked to wear the sensorized t-shirt at all times until the battery ran out, i.e., approximately $18 \mathrm{~h}$. Therefore, there was no need of particular experimental conditions as the patient was free to perform normal activities. As soon as the subject gave the t-shirt back, the data were downloaded and stored in the database for further analysis.

Results: Classifications were performed according to the methodology described in Section III and reported as intrasubject evaluations. Tables III- $X$ show the recognition accuracy in terms of confusion matrices as well as the total average accuracy 
TABLE VII

CONFUSION MATRIX OF SVM CLASSIFIER FOR BP5

\begin{tabular}{|c|c|c|c|}
\hline & Dataset & Depression & Hypomania \\
\hline \multirow{2}{*}{ Depression } & Standard & 85.44 & 14.56 \\
\hline & Markov & 74.87 & 25.13 \\
\hline \multirow{2}{*}{ Hypomania } & Standard & 23.02 & 76.98 \\
\hline & Markov & 3.77 & 96.23 \\
\hline
\end{tabular}

TABLE VIII

CONFUSION MATRIX OF SVM ClASSIFIER FOR BP6

\begin{tabular}{|c|c|c|c|}
\hline & Dataset & Hypomania & Euthymia \\
\hline \hline \multirow{2}{*}{ Hypomania } & Standard & 88.75 & 11.25 \\
\cline { 2 - 4 } & Markov & $\mathbf{9 3 . 9 8}$ & 6.02 \\
\hline \hline \multirow{2}{*}{ Euthymia } & Standard & 27.27 & $\mathbf{7 2 . 7 3}$ \\
\cline { 2 - 4 } & Markov & 7.49 & $\mathbf{9 2 . 5 1}$ \\
\hline
\end{tabular}

Total Accuracies: Standard 80.74\%; Markov 93.24\%. Bold values indicate the best correct classification results for each mood state.

TABLE IX

CONFUSION MATRIX OF SVM CLASSIFIER FOR BP7

\begin{tabular}{|c|c|c|c|}
\hline & Dataset & Depression & Euthymia \\
\hline \hline \multirow{2}{*}{ Depression } & Standard & 96.34 & 3.66 \\
\cline { 2 - 4 } & Markov & $\mathbf{9 7 . 4 7}$ & 2.53 \\
\hline \hline \multirow{2}{*}{ Euthymia } & Standard & 45.56 & 54.44 \\
\cline { 2 - 4 } & Markov & 13.33 & $\mathbf{8 6 . 6 7}$ \\
\hline
\end{tabular}

Total Accuracies: Standard 75.39\%; Markov 92.07\%. Bold values indicate the best correct classification results for each mood state.

TABLE X

CONFUSION MATRIX OF SVM CLASSIFIER FOR BP8

\begin{tabular}{|c|c|c|c|c|}
\hline & Dataset & Mixed-State & Depression & Euthymia \\
\hline \hline \multirow{2}{*}{ Mixed-State } & Standard & 65.86 & 26.42 & 7.72 \\
\cline { 2 - 5 } & Markov & $\mathbf{7 8 . 0 8}$ & 13.70 & 8.22 \\
\hline \hline \multirow{2}{*}{ Depression } & Standard & 13.79 & 78.52 & 7.69 \\
\cline { 2 - 5 } & Markov & 4.51 & $\mathbf{9 3 . 9 0}$ & 1.59 \\
\hline \hline \multirow{2}{*}{ Euthymia } & Standard & 22.60 & 24.66 & 52.74 \\
\cline { 2 - 5 } & Markov & 7.53 & 8.22 & $\mathbf{8 4 . 2 5}$ \\
\hline
\end{tabular}

Total Accuracies: Standard $65.71 \%$; Markov $85.41 \%$. Bold values indicate the best correct classification results for each mood state.

obtained through the LOO procedure on nu-SVMs. Standard dataset refers to an independently processed feature set, i.e., the feature set belonging to a specific acquisition of a patient is taken as an input for the LOO-SVM classification without performing any rescaling procedure. Markov dataset refers to the proposed methodology, i.e., feature set is processed ad hoc in order to consider information from the previous mood state (see details on paragraph III).

According to the description of data processing reported in paragraph III, $n=24$ features constituted the feature space dimension of both standard and Markov datasets. No dimensionality reduction techniques were applied to reduce such a dimension.

Since no patients had a maniac episode, we assigned four labels: hypomania, depression, mixed state, and euthymia.

Patients BP1 and BP3 underwent five visits alongside longterm ANS monitoring. Starting from a period of hypomania, patients reached the ES. As shown in Tables III and IV, considering the proposed Markov dataset, the subjective ANS patterns are well distinguished reaching more than $88 \%$ of accuracy in recognizing the hypomaniac state. Concerning the results obtained from data gathered from BP1, we report that higher classification accuracy on hypomania class was achieved adopting a standard dataset. However, when using the same dataset, high misclassification accuracy was obtained for the euthymia class.

Likewise to BP1 and BP3, patient BP2 began the study showing hypomaniacal psychosis. Then, depressive behaviors were diagnosed changing the mood state to mixed state. Afterwards, the previous observed hypomania state was observed twice. In this case, more than $86 \%$ and $96 \%$ accuracy was reached in distinguishing hypomania from mixed-state patterns.

BP4 experienced depressive psychosis for the whole course of the illness, reaching good affective balance after five monitoring sessions. In order to take into account the unbalanced number of available examples per class, two different learning rates were considered in the SVM training phase, giving the euthymic examples four times more penalty with respect to the depressive examples. As the two considered states are very different in clinical terms, the two patterns resulted strongly distinguished with a recognition accuracy as high as $99.68 \%$.

BP5 showed mood swings between depressive and hypomaniacal psychosis. Despite the fact that about $25 \%$ of the depressive patterns were confused with the hypomaniacal ones, the hypomania states were recognized with more than $96 \%$ accuracy. Likewise for BP1, results obtained on data gathered from BP2 and BP5 show that in using the standard approach, a higher classification accuracy for one class is associated with a very low and insufficient accuracy on another one. Like BP4, patients BP6 and BP7 displayed severe pathological behavior before reaching the euthymic condition. Accordingly, accuracy greater than $86 \%$ was obtained in recognizing such states.

BP8 showed mood swings among three states such as the mixed state, depression, and euthymia. This case is very interesting for this study as we obtained interesting performance, considering a three-class pattern recognition problem (see Table X).

For each patient, higher total accuracy was obtained considering mood states as a Markov chain rather than using a standard approach. Although a further statistical analysis revealed that there are no differences between the sensitivity values given by the two methodologies, significant differences were found concerning the specificity values $(p<0.02)$ and total accuracies $(p<0.01)$ according to the nonparametric Wilcoxon signed rank test for paired data performed on all the subjects. Moreover, in order to generalize these results, it is worthwhile mentioning that we tested the classifier also when data were normalized with respect to a casual mood status achieving also lower performances than the proposed Markovian approach.

\section{CONCLUSION AND Discussion}

In conclusion, we presented a novel wearable system comprised of textile technology and biosignal processing able to recognize the mood status of bipolar patients by means of a personalized hardware and software platform. An effective 
methodology of signal processing based on a mood historydependent, long-term HRV analysis allowed for obtaining robust recognitions validated in a dataset consisting of eight patients who were monitored for up to $18 \mathrm{~h}$ and up to six times, thus collecting more than $400 \mathrm{~h}$ of data. As bipolar disorder is a chronic illness having a high impact on society [40]-[43], research that aims to establish a reliable decision system to support clinical diagnoses is much needed. Physicians, in fact, currently rely on rating scales and questionnaire scores [46]-[48] without any objective clinical clues. Moreover, a typical feature of bipolar disorder is that it is a chronic yet remittent disorder. Patients can experience periods of relative well-being with possible abrupt relapses.

A portable remote system able to detect and predict mood changes will be important for obtaining very useful clinical information even in the absence of direct contact with the physician. This will allow for a more rapid and efficient use of medical treatment and a shortening of the symptomatic period. Although research work reported on biomarkers having statistical difference among different mood states [29], [32], [34]-[36], [49]-[53], a single-variable approach is not sufficient to robustly characterize mood swings [9]. Starting from the consideration that a correlation might exist between mental disorders and ANS dynamical changes, we successfully applied a multiparametric approach as a decision support system for the diagnosis of bipolar disorder. Such information is gathered by means of a wearable, comfortable, and unobtrusive monitoring systems: the PSYCHE platform. ANS signals are acquired over a long term as the mood has been defined as a long-lasting, diffuse, affective state, not associated with a specific trigger [85]. Therefore, no specific tasks are required in using the PSYCHE system. HRV was chosen as a good noninvasive marker of the ANS activity [38], [39], especially effective in emotion recognition systems [70], [86]-[90].

Here, we propose a personalized approach that identifies mood states as intrasubject analysis taking into account the temporal dynamics of the illness. This consideration represents the great novelty of our approach and is implemented considering the ANS dynamics among mood fluctuations as a Markov stochastic process [54], i.e., each mood state refers to the previous one. As literature supports, Markov's theory has been successfully applied to model human emotions [55].

Our methodological approach accounts for clinical course during a mood fluctuation. The onset of a new mood state brings the past clinical history along, and the identification of the next state is more accurate if the previous one is considered. From a point of view purely speculative, our approach goes beyond the rigid application of DSM-IV-TR labels, but introduces the new concept that the clinical diagnosis is looking backward. Better understanding of the patient's mood status can be achieved considering the dynamics of the disorder rather than the single observation treated as completely independent. For instance, patients with bipolar disorders present different clinical signs whether a depressive episode follows or precedes a maniac status [91].

Experimental results are very satisfactory. Considering patients with a mood label such as depression, hypomania, mixed state, and ES, we are able to distinguish subjective mood states with high accuracy, especially when a pathological clinical status is compared with the reference euthymic status (e.g., euthymic versus depressed, and euthymic versus mixed state). Moreover, the comparison of the obtained findings through Markov mood state modeling with a standard approach, inspired by Valenza et al. [9], further confirms the crucial role played by the long-term dynamics of pathological mental states. A further statistical analysis, in fact, revealed that a reliable and significantly higher specificity values are achieved only when the temporal dynamics of the illness is taken into account.The translational clinical application of the proposed methodology is not a challenging task, as ECG monitoring systems are widely available even in a portable fashion (e.g., standard ECG holter). However, as the proposed methodology focuses on the intrasubject classification, the new training phase has to be performed for every new patient. Ideally, such a training phase requires at least four acquisitions of physiological data that cover all the possible mood states, although a minimum switch of two mood states is strongly needed. Moreover, during such a training phase, doctors have to determine the mood states during each of the acquisitions. Then, these labels together with physiological data can be used to train the classifier. Only after this training phase, the proposed system will be able to diagnose the patient without an input from doctors. Major improvements of the system are expected solving the intersubject variability issue for the mood classification.

While the proposed experimental procedure provides for carrying out normal life activities, it is worthwhile mentioning that different life activities could be easily associated with different HRV dynamics. Using the methodology proposed in this study, such changes are minimized considering long-term, historydependent dynamics referred to as one of the four possible pathological mood states. Given the high classification accuracy, it is indeed possible to hypothesize that the few misclassified samples can be interpreted as either algorithmic/mathematical artifacts or physiological outliers, i.e., events not related to mood markers for any reason (including also misleading daily activities).

The impact of this research will open new opportunities to create a closed loop between patients and clinicians through continuous communication and feedback that facilitates disease management by fostering a new collaboration, with more autonomy and empowerment for the patient. Constant monitoring and feedback (to both patients and physicians) are the new keys to managing the illness, to helping patients, to facilitating interaction between patient and physician, as well as to alerting professionals in case of relapse and depressive or manic episodes, and as a ultimate goal, to identifying signal trends in order to predict critical events. Moreover, the possibility of introducing other past mood states to the analysis and mood state labeling features is intriguing also from a clinical point of view. This is because it introduces the idea that mood disorders cannot be considered to be a series of independent and stand-alone states, but rather a chronological sequence of mood states that are related to each other. Previous studies have been limited to not considering this issue and may be one of the possible reasons 
for their lack of ability to discriminate mood status at single subject levels.

Considering mood episodes as interlinked will possibly lead not only to a better characterization of the mood state and a better comprehension of psychophysiology of mood disorders, but also to the possibility of predicting the clinical course of the disorder itself. Further studies with a larger number of recruited patients will be provided a more complete understanding and knowledge of HRV and ANS alterations in bipolar disorders, therefore allowing for the assessment of the most important features related to pathological mental states. Currently, the proposed approach is under development in the framework of the PSYCHE project, which is an European research Project that aims at developing a pervasive and personalized monitoring system for care assessment in mental health.

\section{ACKNOWLEDGMENT}

The authors are grateful to Laura Maley for kindly reviewing the paper. This research leading to these results received support from the European Union Seventh Framework Programme under grant agreement no. 247777 (PSYCHE).

\section{REFERENCES}

[1] E. P. Scilingo, A. Lanatà, and A. Tognetti, "Sensors for wearable systems," in Wearable Monitoring Systems. New York, NY, USA: SpringerVerlag, 2011, pp. 3-25.

[2] A. Lanata, G. Valenza, and E. P. Scilingo, "Eye gaze patterns in emotional pictures," J. Ambient Intell. Humanized Comput., vol. 4, no. 6, pp 705-715, 2013.

[3] R. G. Haahr, S. B. Duun, M. H. Toft, B. Belhage, J. Larsen, K. Birkelund, and E. V. Thomsen, "An electronic patch for wearable health monitoring by reflectance pulse oximetry," IEEE Trans. Biomed. Circuits Syst., vol. 6, no. 1, pp. 45-53, Feb. 2012.

[4] M. Tavakoli, L. Turicchia, and R. Sarpeshkar, "An ultra-low-power pulse oximeter implemented with an energy-efficient transimpedance amplifier," IEEE Trans. Biomed. Circuits Syst., vol. 4, no. 1, pp. 27-38, Feb. 2010.

[5] F. Zhang and Y. Lian, "QRS detection based on multiscale mathematical morphology for wearable ECG devices in body area networks," IEEE Trans. Biomed. Circuits Syst., vol. 3, no. 4, pp. 220-228, Aug. 2009.

[6] E. P. Scilingo, A. Gemignani, R. Paradiso, N. Taccini, B. Ghelarducci, and D. De Rossi, "Performance evaluation of sensing fabrics for monitoring physiological and biomechanical variables," IEEE Trans. Inf. Technol. Biomed., vol. 9, no. 3, pp. 345-352, Sep. 2005.

[7] A. Lanatà, E. P. Scilingo, E. Nardini, G. Loriga, R. Paradiso, and D. DeRossi, "Comparative evaluation of susceptibility to motion artifact in different wearable systems for monitoring respiratory rate," IEEE Trans. Inf. Technol. Biomed., vol. 14, no. 2, pp. 378-386, Mar. 2010.

[8] Y. Chuo, M. Marzencki, B. Hung, C. Jaggernauth, K. Tavakolian, P. Lin, and B. Kaminska, "Mechanically flexible wireless multisensor platform for human physical activity and vitals monitoring," IEEE Trans. Biomed. Circuits Syst., vol. 4, no. 5, pp. 281-294, Oct. 2010.

[9] G. Valenza, C. Gentili, A. Lanatà, and E. P. Scilingo, "Mood recognition in bipolar patients through the psyche platform: Preliminary evaluations and perspectives," Artif. Intell. Med., vol. 57, no. 1, pp. 49-58, 2013.

[10] W. Karlen, C. Mattiussi, and D. Floreano, "Sleep and wake classification with ECG and respiratory effort signals," IEEE Trans. Biomed. Circuits Syst., vol. 3, no. 2, pp. 71-78, Apr. 2009.

[11] D. Zito, D. Pepe, M. Mincica, F. Zito, A. Tognetti, A. Lanata, and D. De Rossi, "SoC CMOS UWB pulse radar sensor for contactless respiratory rate monitoring," IEEE Trans. Biomed. Circuits Syst., vol. 5, no. 6, pp. 503-510, Dec. 2011.

[12] A. Alomainy, Y. Hao, and F. Pasveer, "Numerical and experimental evaluation of a compact sensor antenna for healthcare devices," IEEE Trans. Biomed. Circuits Syst., vol. 1, no. 4, pp. 242-249, Dec. 2007.
[13] A. Lanatà, G. Valenza, and E. P. Scilingo, "A novel EDA glove based on textile-integrated electrodes for affective computing," Med. Biolog. Eng. Comput., vol. 50, no. 11, pp. 1163-1172, 2012.

[14] G. Valenza, A. Lanatà, E. P. Scilingo, and D. De Rossi, "Towards a smart glove: Arousal recognition based on textile electrodermal response," in Proc. IEEE Annu. Int. Conf. Eng. Med. Biol. Soc., 2010, pp. 3598-3601.

[15] C.-T. Lin, C.-J. Chang, B.-S. Lin, S.-H. Hung, C.-F. Chao, and I.-J. Wang, "A real-time wireless brain-computer interface system for drowsiness detection," IEEE Trans. Biomed. Circuits Syst., vol. 4, no. 4, pp. 214-222, Aug. 2010

[16] E. Jovanov, A. Milenkovic, C. Otto, and P. C. De Groen, "A wireless body area network of intelligent motion sensors for computer assisted physical rehabilitation," J. NeuroEng. Rehabil., vol. 2, no. 1, pp. 1-10, 2005

[17] E. D. de Bruin, A. Hartmann, D. Uebelhart, K. Murer, and W. Zijlstra, "Wearable systems for monitoring mobility-related activities in older people: A systematic review," Clin. Rehabil., vol. 22, no. 10-11, pp. 878-895, 2008.

[18] T. Yilmaz, R. Foster, and Y. Hao, "Detecting vital signs with wearable wireless sensors," Sensors, vol. 10, no. 12, pp. 10 837-10 862, 2010.

[19] C. Poon, Q. Liu, H. Gao, W.-H. Lin, and Y.-T. Zhang, "Wearable intelligent systems for e-health," J. Comput. Sci. Eng, vol. 5, no. 3, pp. 246-256, 2011.

[20] S. Patel, H. Park, P. Bonato, L. Chan, and M. Rodgers, "A review of wearable sensors and systems with application in rehabilitation," J. Neuroeng. Rehabil., vol. 9, pp. 1-17, 2012.

[21] A. Pantelopoulos and N. G. Bourbakis, "A survey on wearable sensorbased systems for health monitoring and prognosis," IEEE Trans. Syst., Man, Cybern. C: Appl. Rev., vol. 40, no. 1, pp. 1-12, Jan. 2010.

[22] P. Bonato, "Wearable sensors and systems," IEEE Eng. Med. Biol. Mag., vol. 29, no. 3, pp. 25-36, May/Jun. 2010.

[23] M. Di Rienzo, P. Meriggi, F. Rizzo, P. Castiglioni, C. Lombardi, M. Ferratini, and G. Parati, "Textile technology for the vital signs monitoring in telemedicine and extreme environments," IEEE Trans. Inf. Technol. Biomed., vol. 14, no. 3, pp. 711-717, May 2010.

[24] P. Bonato, "Wearable sensors/systems and their impact on biomedical engineering," IEEE Eng. Med. Biol. Mag., vol. 22, no. 3, pp. 18-20, May/Jun. 2003.

[25] S. Bouwstra, W. Chen, L. Feijs, and S. B. Oetomo, "Smart jacket design for neonatal monitoring with wearable sensors," in Proc. IEEE 6th Int. Workshop Wearable Implantable Body Sens. Netw., 2009, pp. 162-167.

[26] W. Chen, J. Hu, S. Bouwstra, and S. B. Oetomo, "Sensor integration for perinatology research," Int. J. Sens. Netw., vol. 9, no. 1, pp. 38-49, 2011.

[27] G. Valenza, A. Lanatà, M. Ferro, and E. P. Scilingo, "Real-time discrimination of multiple cardiac arrhythmias for wearable systems based on neural networks," in Proc. Computers in Cardiology, 2008, pp. 1053-1056.

[28] B. Levy, "Autonomic nervous system arousal and cognitive functioning in bipolar disorder," Bipolar Disorders, vol. 15, no. 1, pp. 70-79, 2013.

[29] K. Latalova, J. Prasko, T. Diveky, A. Grambal, D. Kamaradova, H. Velartova, J. Salinger, and J. Opavsky, "Autonomic nervous system in euthymic patients with bipolar affective disorder," Neuro Endocrinol. Lett., vol. 31, no. 6, pp. 829-836, 2010.

[30] B. L. Henry, A. Minassian, M. P. Paulus, M. A. Geyer, and W. Perry, "Heart rate variability in bipolar mania and schizophrenia," J. Psychiatr. Res., vol. 44, no. 3, pp. 168-176, 2010.

[31] A. Voss, V. Baier, S. Schulz, and K. Bar, "Linear and nonlinear methods for analyses of cardiovascular variability in bipolar disorders," Bipolar Disorders, vol. 8, no. 5p1, pp. 441-452, 2006.

[32] A. Fagiolini, K. Chengappa, I. Soreca, and J. Chang, "Bipolar disorder and the metabolic syndrome: causal factors, psychiatric outcomes and economic burden," CNS Drugs, vol. 22, no. 8, pp. 655-669, 2008.

[33] E. Ruiz-Padial, J. Vila, and J. F. Thayer, "The effect of conscious and non-conscious presentation of biologically relevant emotion pictures on emotion modulated startle and phasic heart rate," Int. J. Psychophysiol., vol. 79, no. 3, pp. 341-346, 2011.

[34] R. Carney, K. Freedland, M. Rich, and A. Jaffe, "Depression as a risk factor for cardiac events in established coronary heart disease: A review of possible mechanisms," Ann. Behav. Med., vol. 17, no. 2, pp. 142-149, 1995.

[35] A. Glassman, "Depression, cardiac death, and the central nervous system," Neuropsychobiology, vol. 37, no. 2, pp. 80-83, 1998.

[36] L. Watkins, J. Blumenthal, and R. Carney, "Association of anxiety with reduced baroreflex cardiac control in patients after acute myocardial infarction," Amer. Heart J., vol. 143, no. 3, pp. 460-466, 2002.

[37] D. Christmas, J. D. Steele, S. Tolomeo, M. S. Eljamel, and K. Matthews, "Vagus nerve stimulation for chronic major depressive disorder: 12-month 
outcomes in highly treatment-refractory patients," J. Affect. Disorders, vol. 150, no. 3, pp. 1221-1225, 2013

[38] A. Camm, M. Malik, J. Bigger, G. Breithardt, S. Cerutti, R. Cohen, Task Force of the European Society of Cardiology, and the North American Society of Pacing and Electrophysiology, "Heart rate variability: Standards of measurement, physiological interpretation, and clinical use," Circulation, vol. 93, no. 5, pp. 1043-1065, 1996

[39] U. Rajendra Acharya, K. Paul Joseph, N. Kannathal, C. Lim, and J. Suri, "Heart rate variability: A review," Med. Biolog. Eng. Comput., vol. 44, no. 12, pp. 1031-1051, 2006.

[40] R. C. Kessler, K. A. McGonagle, S. Zhao, C. B. Nelson, M. Hughes, S. Eshleman, H. U. Wittchen, and K. S. Kendler, "Lifetime and 12-month prevalence of dsm-iii-r psychiatric disorders in the united states: Results from the national comorbidity survey," Arch. General Psychiatry, vol. 51, no. 1, p. 8, 1994.

[41] H. Wittchen and F. Jacobi, "Size and burden of mental disorders in europe-A critical review and appraisal of 27 studies," Eur. Neuropsychopharmacol., vol. 15, no. 4, pp. 357-376, 2005.

[42] S. Pini, V. de Queiroz, D. Pagnin, L. Pezawas, J. Angst, G. B. Cassano, and H. U. Wittchen, "Prevalence and burden of bipolar disorders in european countries," Eur. Neuropsychopharmacol., vol. 15, no. 4, pp. 425-434, 2005.

[43] Y. Chen and S. Dilsaver, "Lifetime rates of suicide attempts among subjects with bipolar and unipolar disorders relative to subjects with other axis i disorders," Biological Psychiatry, vol. 39, no. 10, pp. 896-899, 1996.

[44] K. R. Merikangas, R. Jin, J. P. He, R. C. Kessler, S. Lee, N. A. Sampson, M. C. Viana, L. H. Andrade, C. Hu, E. G. Karam, M. Ladea, M. E. Medina-Mora, Y. Ono, J. Posada-Villa, R. Sagar, J. E. Wells, and Z. Zarkov, "Prevalence and correlates of bipolar spectrum disorder in the world mental health survey initiative," Arch. General Psychiatry, vol. 68, no. 3, pp. 241-251, 2011.

[45] A. P. Association, Diagnostic and Statistical Manual of Mental Disorders: DSM-IV-TR. Arlington, VA, USA: American Psychiatric Publishing, Inc., 2000.

[46] E. Vieta, M. Reinares, and A. Rosa, "Staging bipolar disorder," Neurotoxicity Res., vol. 19, no. 2, pp. 279-285, 2011.

[47] A. Andreazza, M. Kauer-Sant'Anna, B. Frey, D. Bond, F. Kapczinski, L. Young, and L. Yatham, "Oxidative stress markers in bipolar disorder: A meta-analysis," J. Affective Disorders, vol. 111, no. 2, pp. 135-144, 2008.

[48] M. Phillips and E. Vieta, "Identifying functional neuroimaging biomarkers of bipolar disorder: Toward dsm-v," Schizophrenia Bull., vol. 33, no. 4, pp. 893-904, 2007.

[49] H. Stampfer, "The relationship between psychiatric illness and the circadian pattern of heart rate," Australasian Psychiatry, vol. 32, no. 2, pp. 187-198, 1998.

[50] G. Iverson, H. Stampfer, and M. Gaetz, "Reliability of circadian heart pattern analysis in psychiatry," Psychiatr. Quart., vol. 73, no. 3, pp. 195 203, 2002

[51] G. Iverson, M. Gaetz, E. Rzempoluck, P. McLean, W. Linden, and R. Remick, "A new potential marker for abnormal cardiac physiology in depression," J. Behavioral Med., vol. 28, no. 6, pp. 507-511, 2005.

[52] J. Taillard, P. Sanchez, P. Lemoine, and J. Mouret, "Heart rate circadian rhythm as a biological marker of desynchronization in major depression: A methodological and preliminary report," Chronobiol. Int., vol. 7, no. 4, pp. 305-316, 1990.

[53] J. Taillard, P. Lemoine, P. Boule, M. Drogue, and J. Mouret, "Sleep and heart rate circadian rhythm in depression: The necessity to separate,' Chronobiol. Int., vol. 10, no. 1, pp. 63-72, 1993.

[54] C. W. Gardine, Handbook of Stochastic Methods. vol. 3, Berlin, Germany: Springer-Verlag, 1985.

[55] A. Chandra, "A computational architecture to model human emotions," in Proc. Intelligent Information Systems, 1997, pp. 86-89.

[56] A. Greco, A. Lanata, G. Valenza, G. Rota, N. Vanello, and E. Scilingo, "On the deconvolution analysis of electrodermal activity in bipolar patients,' in Proc. IEEE Annu. Int. Conf. Eng. Med. Biol. Soc, 2012, pp. 6691-6694.

[57] N. Vanello, A. Guidi, C. Gentili, S. Werner, G. Bertschy, G. Valenza, A. Lanata, and E. P. Scilingo, "Speech analysis for mood state characterization in bipolar patients," in Proc. IEEE Annu. Int. Conf. Eng. Med. Biol. Soc., 2012, pp. 2104-2107.

[58] S. Mariani, M. Migliorini, G. Tacchino, C. Gentili, G. Bertschy, S. Werner, and A. M. Bianchi, "Clinical state assessment in bipolar patients by means of HRV features obtained with a sensorized t-shirt," in Proc. IEEE Annu. Int. Conf. Eng. Med. Biol. Soc., 2012, pp. 2240-2243.
[59] J. G. Webste, Medical Instrumentation: Application and Design.. New York: Wiley, 1998

[60] U. R. Acharya, K. P. Joseph, N. Kannathal, C. Lim, and J. Suri, "Heart rate variability: A review," Med. Biolog. Eng. Comput., vol. 44, no. 12, pp. 1031-1051, 2006.

[61] J. Pan and W. Tompkins, "A real-time QRS detection algorithm," IEEE Trans. Biomed. Eng., vol. BME-32, no. 3, pp. 230-236, Mar. 1985.

[62] H. Akaike, "Fitting autoregressive models for prediction," Ann. Inst. Statist. Math., vol. 21, no. 1, pp. 243-247, 1969.

[63] F. Atyabi, M. Livari, K. Kaviani, and M. Tabar, "Two statistical methods for resolving healthy individuals and those with congestive heart failure based on extended self-similarity and a recursive method," J. Biological Phys., vol. 32, no. 6, pp. 489-495, 2006.

[64] L. Glass, "Introduction to controversial topics in nonlinear science: Is the normal heart rate chaotic?," Chaos: Interdiscipl. J. Nonlinear Sci., vol. 19 no. 2, p. $028501,2009$.

[65] L. Glass, "Synchronization and rhythmic processes in physiology," $\mathrm{Na}$ ture, vol. 410, no. 6825, pp. 277-284, 2001.

[66] A. Goldberger, C. Peng, and L. Lipsitz, "What is physiologic complexity and how does it change with aging and disease?," Neurobiol. aging, vol. 23, no. 1, pp. 23-26, 2002.

[67] C. Poon and C. Merrill, "Decrease of cardiac chaos in congestive heart failure," Nature, vol. 389, no. 6650, pp. 492-495, 1997.

[68] M. P. Tulppo, A. M. Kiviniemi, A. J. Hautala, M. Kallio, T. H. Seppänen, T. Mäkikallio, and H. V. Huikuri, "Physiological background of the loss of fractal heart rate dynamics," Circulation, vol. 112, no. 3, p. 314, 2005.

[69] G. Wu, N. M. Arzeno, L. L. Shen, D. K. Tang, D. A. Zheng, N. Q. Zhao, D. L. Eckberg, and C. S. Poon, "Chaotic signatures of heart rate variability and its power spectrum in health, aging and heart failure," PloS one, vol. 4, no. 2, p. e4323, 2009.

[70] G. Valenza, A. Lanata, and E. P. Scilingo, "The role of nonlinear dynamics in affective valence and arousal recognition," IEEE Trans. Affective Computing, vol. 3, no. 2, pp. 237-249, Apr.-Jun. 2012.

[71] G. Valenza, A. Lanata, and E. P. Scilingo, "Oscillations of heart rate and respiration synchronize during affective visual stimulation," IEEE Trans. Inf. Technol. Biomed., vol. 16, no. 4, pp. 683-690, Jul. 2012.

[72] G. Valenza, L. Citi, E. Scilingo, and R. Barbieri, "Point-process nonlinear models with laguerre and volterra expansions: Instantaneous assessment of heartbeat dynamics," IEEE Trans. Signal Process., vol. 61, no. 11, pp. 2914-2926, Jun. 2013.

[73] J. Theiler, "Efficient algorithm for estimating the correlation dimension from a set of discrete points," Phys. Rev. A, vol. 36, no. 9, pp. 4456-4462, 1987.

[74] Y. Fusheng, H. Bo, and T. Qingyu, "Approximate Entropy and its application in biosignal analysis," Nonlinear Biomed. Signal Process., vol. 2, pp. 72-91, 2000.

[75] J. Richman and J. Moorman, "Physiological time-series analysis using approximate entropy and sample entropy," Amer. J. Physiol.-Heart Circulat. Physiol., vol. 278, no. 6, pp. H2039-H2049, 2000.

[76] N. Marwan, M. Carmen Romano, M. Thiel, and J. Kurths, "Recurrence plots for the analysis of complex systems," Phys. Rep., vol. 438, no. 5-6, pp. 237-329, 2007.

[77] J. Zbilut and C. Webber Jr., Recurrence Quantification Analysis. New York: Wiley Online Library, 2006.

[78] C. Peng, S. Buldyrev, S. Havlin, M. Simons, H. Stanley, and A. Goldberger, "Mosaic organization of DNA nucleotides," Phys. Rev. E, vol. 49, no. 2, pp. 1685-1690, 1994.

[79] C. Peng, S. Havlin, H. Stanley, and A. Goldberger, "Quantification of scaling exponents and crossover phenomena in nonstationary heartbeat time series," Chaos interdisciplinary J. Nonlinear Sci., vol. 5, no. 1, pp. 8287, 1995.

[80] M. Kearns and D. Ron, "Algorithmic stability and sanity-check bounds for leave-one-out cross-validation," Neural Comput., vol. 11, no. 6, pp. 1427 1453, 1999.

[81] B. Schölkopf, A. J. Smola, R. C. Williamson, and P. L. Bartlett, "New support vector algorithms," Neural Comput., vol. 12, no. 5, pp. 1207$1245,2000$.

[82] R. Kohavi and F. Provost, "Glossary of terms," Mach. Learn., vol. 30 no. June, pp. 271-274, 1998.

[83] C.-C. Chang and C.-J. Lin. (2011). LIBSVM: A library for support vector machines. ACM Trans. Intell. Syst. Technol. [Online]. 2, pp. 27:1-27:27. Software Available:http://www.csie.ntu.edu.tw/ cjlin/libsvm

[84] A. Schlogl, time Series Analysis-A toolbox for the use with Matlab, [Online]. Available: http://pub.ist.ac.at/\%7Eschloegl/matlab/tsa/ (Accessed: 3 Dec. 2012) 
[85] S. Johnson, J. Gruber, and L. Eisner, "Emotion and bipolar disorder," in Emotion and Psychopathology.. Washington, DC, USA: American Psychological Assoc., 2007.

[86] L. Ivonin, H.-M. Chang, W. Chen, and M. Rauterberg, "Automatic recognition of the unconscious reactions from physiological signals," in Human Factors in Computing and Informatics. New York, NY, USA: SpringerVerlag, 2013, pp. 16-35.

[87] L. Ivonin, H.-M. Chang, W. Chen, and M. Rauterberg, "Unconscious emotions: quantifying and logging something we are not aware of," Pers. Ubiquitous Comput., vol. 17, no. 4, pp. 663-673, 2013.

[88] R. A. Calvo and S. D’Mello, "Affect detection: An interdisciplinary review of models, methods, and their applications," IEEE Trans. Affective Comput., vol. 1, no. 1, pp. 18-37, Jan. 2010

[89] G. Valenza, A. Lanatá, and E. P. Scilingo, "Improving emotion recognition systems by embedding cardiorespiratory coupling," Physiol. Meas., vol. 34, no. 4, pp. 449-464, 2013.

[90] G. Valenza, P. Allegrini, A. Lanatà, and E. P. Scilingo, "Dominant lyapunov exponent and approximate entropy in heart rate variability during emotional visual elicitation," Frontiers Neuroeng., vol. 5, pp. 1-7, 2012.

[91] A. Koukopoulos, D. Reginaldi, L. Tondo, C. Visioli, and R. Baldessarini, "Course sequences in bipolar disorder: Depressions preceding or following manias or hypomanias," J. Affect. Disorders, vol. 151, pp. 105-110, 2013.

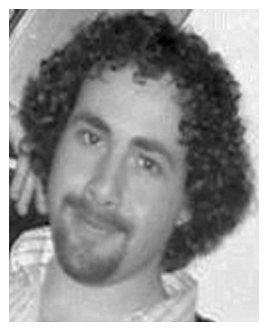

Gaetano Valenza (S'10-M'12) received the Ph.D. degree in automation, robotics, and bioengineering from the University of Pisa, Pisa, Italy, in 2013.

$\mathrm{He}$ is currently a Postdoctoral Fellow with the Department of Information Engineering and the Research Center "E. Piaggio," University of Pisa. The main topics of his research are biomedical signal processing, cardiovascular and neural modeling, and wearable systems for physiological monitoring. Applicative fields include affective computing, assessment of mood disorders, and characterization of

disorder of consciousness.

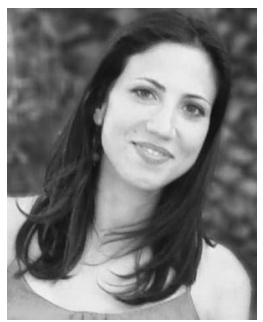

Mimma Nardelli received the Bachelor's and Master's degrees in biomedical engineering from the University of Pisa, Pisa, Italy, in 2009 and 2012, respectively. She is currently working toward the Ph.D. degree at the University of Pisa and is with the Research Center "E. Piaggio."

Her research interests are mainly related to the study of nonlinear dynamics in physiological signals gathered from the central and autonomic nervous systems. Her fields of application include affective computing, assessment of mood disorders, and disorders

of consciousness.

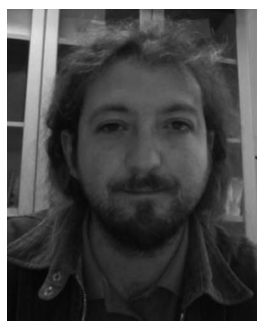

Antonio Lanatà (M'12) received the graduate degree in electronic engineering in 2001. He received the Ph.D. degree in automation, robotics and bioengineering from the University of Pisa, Pisa, Italy, in 2006.

$\mathrm{He}$ is with the Department of Information Engineering and with the Research Centre E. Piaggio, Faculty of Engineering, University of Pisa, Italy. His research interests are focused on developing Ultra wideband wearable systems for biomedical applications and high-level signals processing. Currently, the main fields of application are affective computing, mental and consciousness disorders. He has published numerous articles in international scientific journals and an author of several chapters of books.

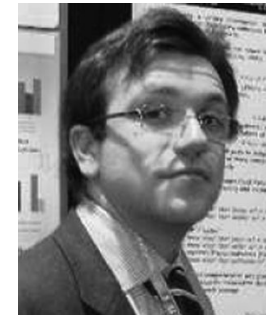

Claudio Gentili received the M.D. and Ph.D. degrees from the University of Pisa, Italy, in 2002 and 2007, respectively, and the M.S. degree in psychology from the University of Pavia, Italy, in 2009.

$\mathrm{He}$ is currently an Assistant Professor in clinical psychology at Pisa University, Pisa, Italy. His main fields of research are neurobiology of psychological and personality traits, neurobiology and psychophysiology of anxiety and mood disorders. In particular, he is interested in neurobiology of social anxiety and social phobia using fMRI facilities.

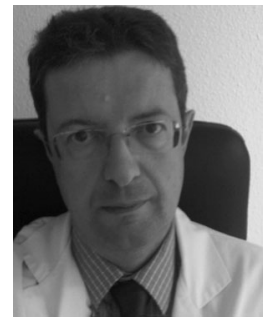

chopharmacology.
Gilles Bertschy was born in 1961. He did his clinical training in Besanon, France, and received the Ph.D. degree in psychopharmacology at Paris-6 University, Paris, France. He is a Professor of adult psychiatry in Strasbourg University, Strasbourg, France, and the head of a division in the Strasbourg University Hospital. His career lead him to work in the university departments of psychiatry in Cotonou, Benin and Lausanne and Geneva, Switzerland. He is a Specialist of mood disorders, he developed research activities in the fields of clinics, biological markers and psy-

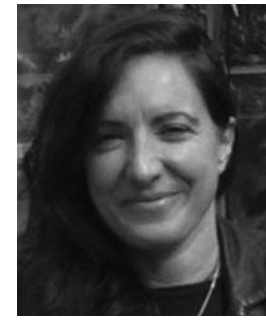

Rita Paradiso received the graduate degree in physics from the University of Genoa, Genoa, Italy, and the Ph.D. degree in bioengineering in 1991.

She joined Smartex in 2000 as a R\&D Manager, and from July 2011 she is the CEO of the company. She has gained experience in management and coordination of European projects. Her main research topis include molecular electronics, biosensors, biomaterials for biomedical applications and e-textile. She has more than 60 scientific publications and conference presentation since 1989 .

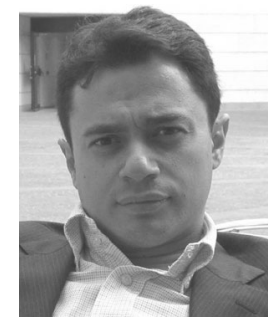

Enzo Pasquale Scilingo (M'10) received the Laurea degree in electronic engineering from the University of Pisa, Pisa, Italy, and the Ph.D. degree in bioengineering from the University of Milan, MIlan, Italy, in 1995 and 1998, respectively.

$\mathrm{He}$ is currently an Associate Professor with the University of Pisa and carries out his research activity at the Information Engineering Department, where he leads the laboratory Biolab and at the Research Center "E. Piaggio." He is currently coordinating a European project EC-FP7-ICT-247777 "PSYCHEPersonalised monitoring SYstems for Care in mental Health.' 